\title{
NGC 1566: Spectroscopy of a symmetric system with Seyfert nucleus ${ }^{\star}$
}

\author{
E. L. Agüero ${ }^{1, \star \star}$, R. J. Díaz ${ }^{1,2}$, and E. Bajaja ${ }^{3, \star \star}$ \\ ${ }^{1}$ Observatorio Astronómico, Universidad Nacional de Córdoba, Laprida 854, 5000 Córdoba, Argentina \\ e-mail: aguero@oac.uncor.edu \\ 2 SECyT, Universidad Nacional de Córdoba \\ e-mail: diaz@oac.uncor.edu \\ 3 Instituto Argentino de Radioastronomía, CC 5, 1894 Villa Elisa, Prov. de Bs. As., Argentina \\ e-mail: bajaja@conae.gov.ar
}

Received 28 May 2003 / Accepted 11 August 2003

\begin{abstract}
The central region and main body of the very symmetrical galaxy NGC 1566 were observed and studied spectrophotometrically and kinematically. We found that: a) the total masses derived from the Satoh model fitted to the rotation curve, and from the global $\mathrm{H}$ I radial velocity profile, are coincident: $2 \times 10^{11} M_{\odot}$, which implies the presence of two galactic subsystems without the need for a massive halo; b) from the rotation curve of the fitted model an outflow motion at the bar tips is confirmed $\left(\Delta V \sim 40 \mathrm{~km} \mathrm{~s}^{-1}\right)$; c) the observed non-circular motions in some regions with significant blue shifts $\left(\Delta V \sim 60 \mathrm{~km} \mathrm{~s}^{-1}\right)$ would represent inflows of gas to the nucleus; d) the fitted pattern speed at $(23 \pm 2) \mathrm{km} \mathrm{s}^{-1} \mathrm{kpc}^{-1}$ allows one to explain the radial positions of the inner ring, the star formation ridge of the arms, and the ring at the main disk outer edge, as result of dynamical resonances; e) the nucleus was in a stage of Seyfert 1.8 and in a low level of activity during observations; f) nuclear and inner rings were detected; there is also evidence of a ring at the main disk outer edge; g) some emission regions in the arms have $\mathrm{H} \alpha$ fluxes higher than in the nucleus; h) the star bursts in the ring at the bar end radius would be younger than in the arms; i) the $\mathrm{H} \alpha$ emission appears to be correlated with the thermal component of the continuum radiation and with the molecular gas over the whole galaxy.
\end{abstract}

Key words. galaxies: individual: NGC 1566 - galaxies: photometry - galaxies: nuclei - galaxies: kinematics and dynamics

\section{Introduction}

NGC 1566, the brightest member of the Doradus Group, is an $\mathrm{SAB}(\mathrm{s}) \mathrm{bc}$ galaxy that presents a symmetrical optical structure, with a prominent system of very regular arms that emerge from the ends of a little central bar with its major axis along PA $\sim 5^{\circ}$. The galaxy does not present important photometric morphological distortions (see the HST images by Malkan et al. 1998) at circumnuclear scales. Its nucleus is a Seyfert type one that shows photometric variability. It was proposed in general that the nuclear activity could originate in strong deviations of the galactic gravitational potential from axial symmetry (e.g. Combes 2001). Due to the high degree of symmetry that this

Send offprint requests to: R. J. Díaz,

e-mail: diaz@oac.uncor.edu

* Paper partially supported by a grant from SeCyT, National University of Córdoba, Argentina.

$\star \star$ Visiting astronomers at the Complejo Astronómico El Leoncito (CASLEO) operated under agreement between the Consejo Nacional de Investigaciones Científicas y Técnicas de la República Argentina and the National Universities of La Plata, Córdoba and San Juan. object presents, the only morphological subsystem that could generate gas inflows is the small central bar.

NGC 1566 is studied here spectroscopically to improve the general knowledge of its nature, making a detailed analysis of the emissive and kinematical properties of the most conspicuous morphological features of its central region and main body. The study was based on a set of intermediate resolution spectra taken with a long slit at six different position angles through the nucleus, the results of which are compared with observations at radio frequencies.

\section{Observations and reductions}

Spectroscopic observations of NGC 1566 were performed on November 9 and 10, 1996, with a REOSC spectrograph attached to the $2.15 \mathrm{~m}$ Ritchey-Chrétien telescope of the Complejo Astronómico EL Leoncito (San Juan, Argentina). The detector was a Tektronix CCD $1024 \times 1024$ pixels and the spectra were obtained with a slit of $22^{\prime \prime} 8 \times 348^{\prime \prime}$, at the six position angles indicated in Fig. 1. These position angles were selected according to previous results based on the CO emission line observations by Bajaja et al. (1995) and the 


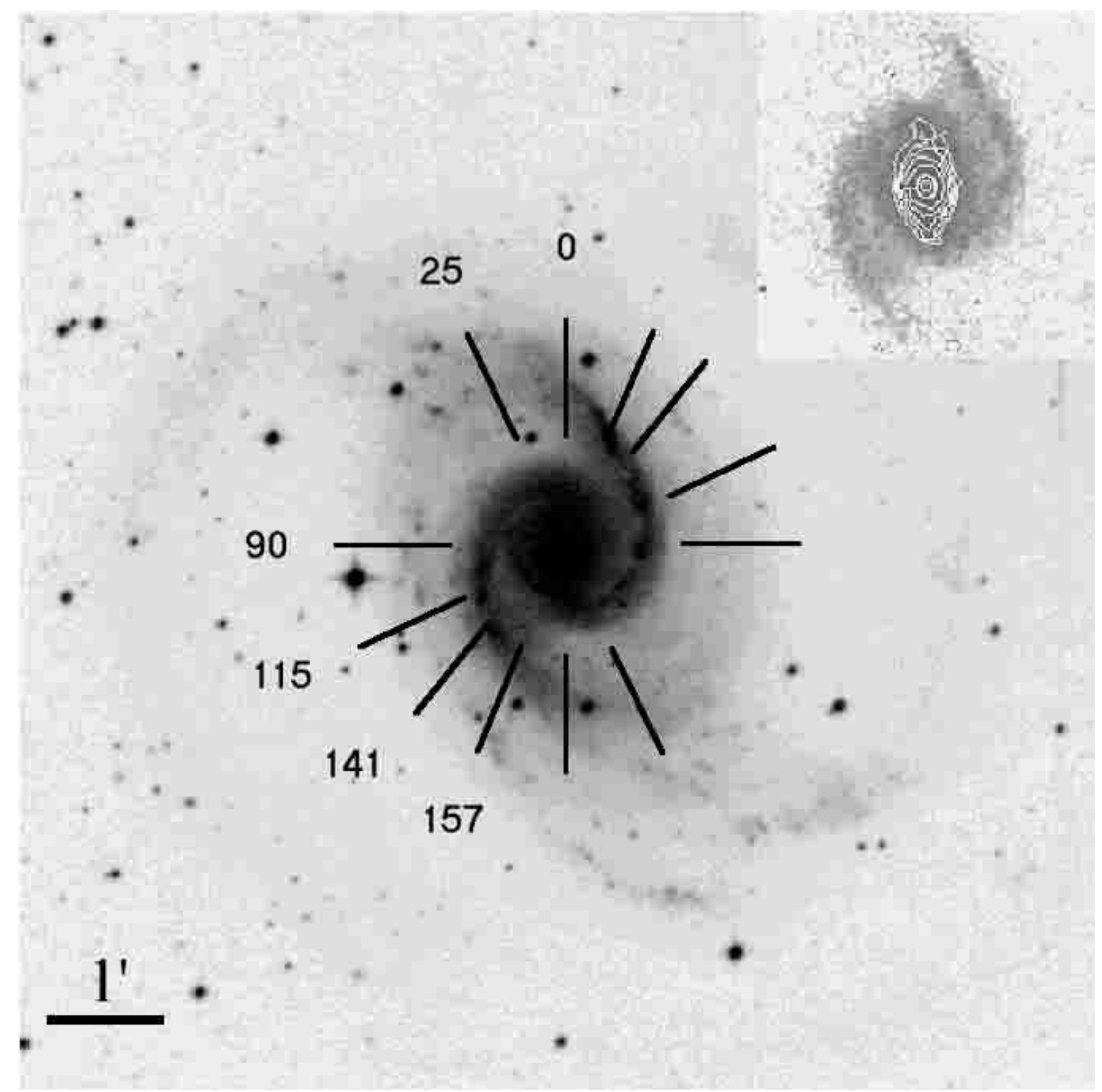

Fig. 1. Blue image (from ESO-DSS plates) of NGC 1566. North is at the top and East to the left. The lines represent the position angles of the slit. Upper right corner: central region of the galaxy at $H$-band (from 2-MASS), processed to show the structure of the bar.

apparent distribution of $\mathrm{HII}$ regions in the galaxy. Three frames, with a total exposure of $45 \mathrm{~min}$, were taken at each position. A 1200 lines $\mathrm{mm}^{-1}$ grating was used, covering the wavelength range of $\lambda \lambda 6200-6900$. The dispersion was $32 \AA \mathrm{mm}^{-1}$, the reciprocal dispersion $0.76 \AA$ pixel $^{-1}$, the resolution $2.5 \AA$, and the angular scale $1^{\prime \prime} .02$ pixel $^{-1}$. The seeing during the observations was about 2 '. 5 . The spectra were flux calibrated with stars from the Stone \& Baldwin (1982) catalog. The usual procedure was followed for the observations including the registrations of the bias, the flat field, the dark current, and the comparison spectra. Data reduction was carried out using standard methods in the IRAF (developed by NOAO) reduction package.

\section{Results}

\subsection{Radial velocities}

Radial velocities of NGC 1566 were derived from the obtained spectra by measuring the centroids of the Gaussian curves fitted to the profiles of the $\mathrm{H} \alpha$ and the [N II] $\lambda \lambda 6548,6584$ emission lines. The signal to noise ratios $(S / N)$ for the $\mathrm{H} \alpha$ emission line is $S / N \sim 50$ at the nuclear region, $S / N \sim 120$ at the giant $\mathrm{H}$ II regions in the arms and $S / N \sim 3$ at the faintest outer regions with determined radial velocity. Considering the symmetry of the observed velocity curves, the adopted systemic heliocentric radial velocity is $V=(1508 \pm 10) \mathrm{km} \mathrm{s}^{-1}$, coincident, within the error bars, with the velocity determined at radio frequencies by Bajaja et al. (1995). Referred to the galactic standard of rest, that velocity becomes $V_{0}=1335 \mathrm{~km} \mathrm{~s}^{-1}$ which leads to a distance of $17.8 \mathrm{Mpc}\left(H_{0}=75 \mathrm{~km} \mathrm{~s}^{-1} \mathrm{Mpc}^{-1}\right)$ and to a scale of $86 \mathrm{pc}\left({ }^{\prime \prime}\right)^{-1}$.

An axial symmetric rotation model from a double component Satoh density law (Binney 1994) was fitted to the whole set of data. The best geometrical parameters derived were $i=(35 \pm 3)^{\circ}, \mathrm{PA}_{0}=(44 \pm 8)^{\circ}$. The rotation curve corresponding to this model, with the deprojected velocity measurements in the three position angles nearest to the major axis, is 


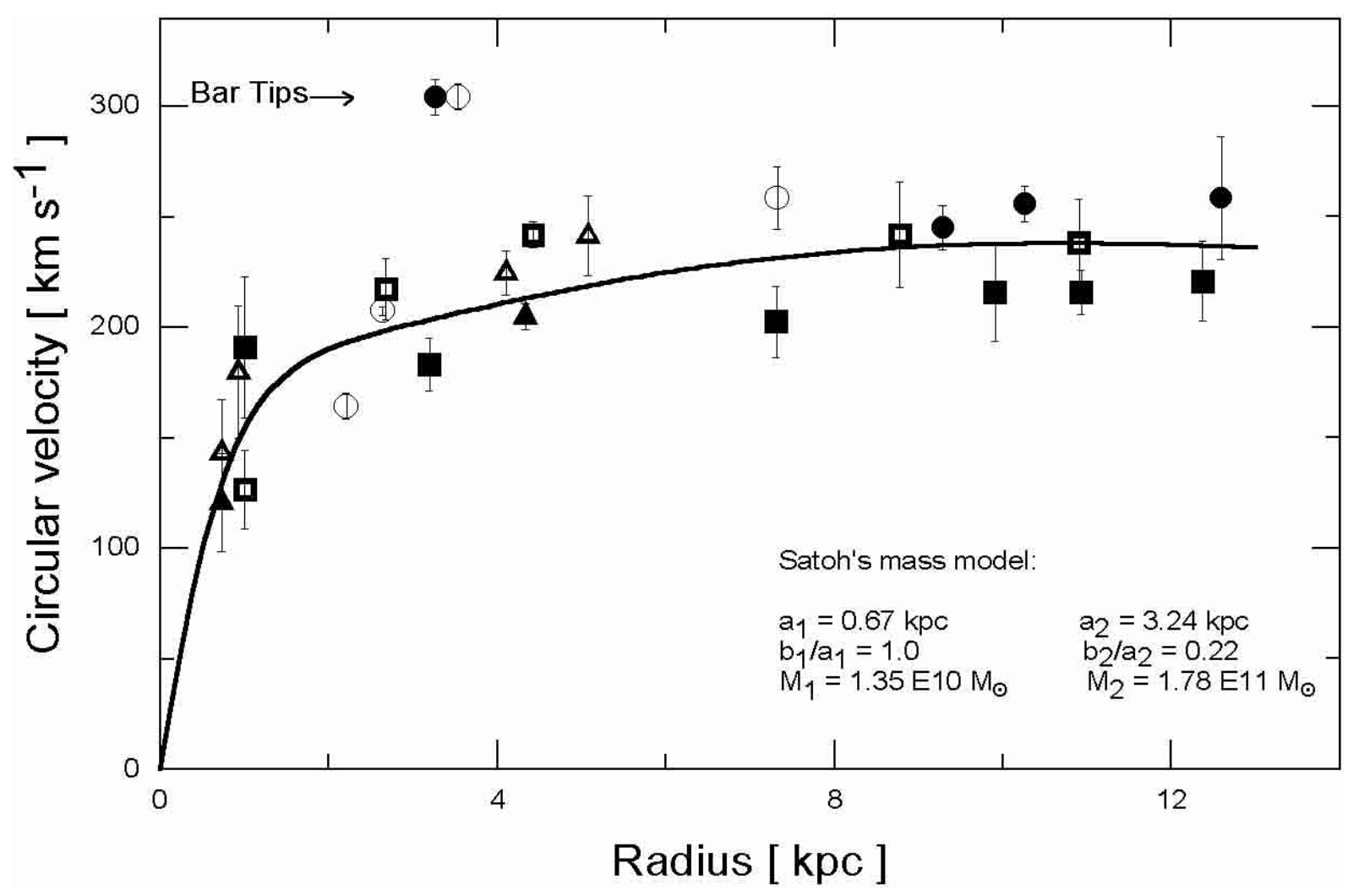

Fig. 2. Rotation curve of the fitted Satoh model, with the deprojected velocity measurements along the position angles nearest to the derived kinematical major axis: $\mathrm{PA}=0^{\circ}$ (circles), $\mathrm{PA}=25^{\circ}$ (boxes) and $\mathrm{PA}=90^{\circ}$ (triangles). Filled symbols correspond to the near side of the galaxy. Note that the ionized gas at the bar tips does not follow the fitted model.

presented in Fig. 2; a rotation velocity of $230 \mathrm{~km} \mathrm{~s}^{-1}$ is reached at a distance of $140^{\prime \prime}$, the largest distance for an optical measurement on this galaxy to date. The Keplerian mass within this radius is about $1.5 \times 10^{11} M_{\odot}$; if the curve remains flat up to the external optical radius $\left(R_{25} \approx 330^{\prime \prime}\right)$, the resulting mass would be about $3 \times 10^{11} M_{\odot}$. The maximum rotation velocity is similar to the normal values in Sbc galaxies $\left(\sim 200 \mathrm{~km} \mathrm{~s}^{-1}\right.$, Rubin et al. 1985 and references therein). The parameters of the fitted Satoh components, shown in Fig. 2, imply the presence of only two galactic subsystems similar to an intermediate bulge-disk and a disk, and a total mass of $1.92 \times 10^{11} M_{\odot}$. The global H I profile (Reif et al. 1982) has a width, at a $20 \%$ level, of $(233 \pm 10) \mathrm{km} \mathrm{s}^{-1}$. The method described in Tully (1988) was used here to derive a rotational kinematical width $\left(W_{R}\right)$, which allows an estimation of the total mass of the galaxy through $M_{T}^{\mathrm{HI}}=\left(W_{\mathrm{R}} / \sin i\right)^{2} \Delta_{25}^{b, i} / 8 G$, where $G$ is the universal gravitational constant and $\Delta_{25}^{b, i}$ is the optical diameter at $R_{25}(31.3 \mathrm{kpc}, h=0.75$; Tully 1988$)$. The obtained value, $M_{T}^{\mathrm{HI}}=1.96 \times 10^{11} M_{\odot}$, is very close to that derived from the Satoh model. Parkes $21 \mathrm{~cm}$ observations (Becker et al. 1988) indicate that the H I extends to up to $90 \%$ of the optical disk, which also points to a not extended halo and is consistent with the representation of the rotation curve by two galactic subsystems, without the need for a halo component.

Two points at the bar tips on $\mathrm{PA}=0^{\circ}$ do not fit the rotation model, which implies, assuming a trailing spiral pattern, outflow motion near the bar major axis with a minimum velocity of $40 \mathrm{~km} \mathrm{~s}^{-1}$, which is the observed excess in radial velocity.
This outflow motion was also detected in a smaller set of radial velocities presented by Beckmann et al. (1986), although they determined it from a somewhat different set of geometrical parameters $\left(i=30^{\circ}, \mathrm{PA}_{0}=20^{\circ}\right)$. Inflow motion is detected along $\mathrm{PA}=115^{\circ}$ (Fig. 3), near to the minor axis of the system, revealing the presence of non-circular motions, reaching a value of about $60 \mathrm{~km} \mathrm{~s}^{-1}$ towards the line of sight, at a region $10^{\prime \prime}$ to the ESE (far side) of the nucleus (which corresponds to high $S / N$ detections). From the locations of these regions, and assuming that those motions take place on the galactic plane, they would represent inflows of gas towards the nucleus. The small bar seen in the central region being the only deviation observed from axial symmetry, these inflows could constitute the mechanism to feed the active nucleus of NGC 1566. The construction of a detailed dynamical model would make it possible to conclude if the low luminosity bar of NGC 1566 has enough gravitational potential to generate those non-circular motions (Athanassoula 1992; Piner et al. 1995).

The angular velocity curve corresponding to the fitted axial symmetric model is illustrated in Fig. 4, as well as the Lindblad $\Omega-\kappa / 2$ and the ultra-harmonic $\Omega-\kappa / 4$ curves. If the corotation resonance is located at the ring of the main disk outer edge, only one solution of the perturbing pattern speed fits the most important features marked in Figs. 7-9. A pattern angular velocity of $(23 \pm 2) \mathrm{km} \mathrm{s}^{-1} \mathrm{kpc}^{-1}$ represents a unique solution that explains the main morphological features observed and pointed out in these figures. From the optical morphology, Elmergreen \& Elmergreen (1990) 


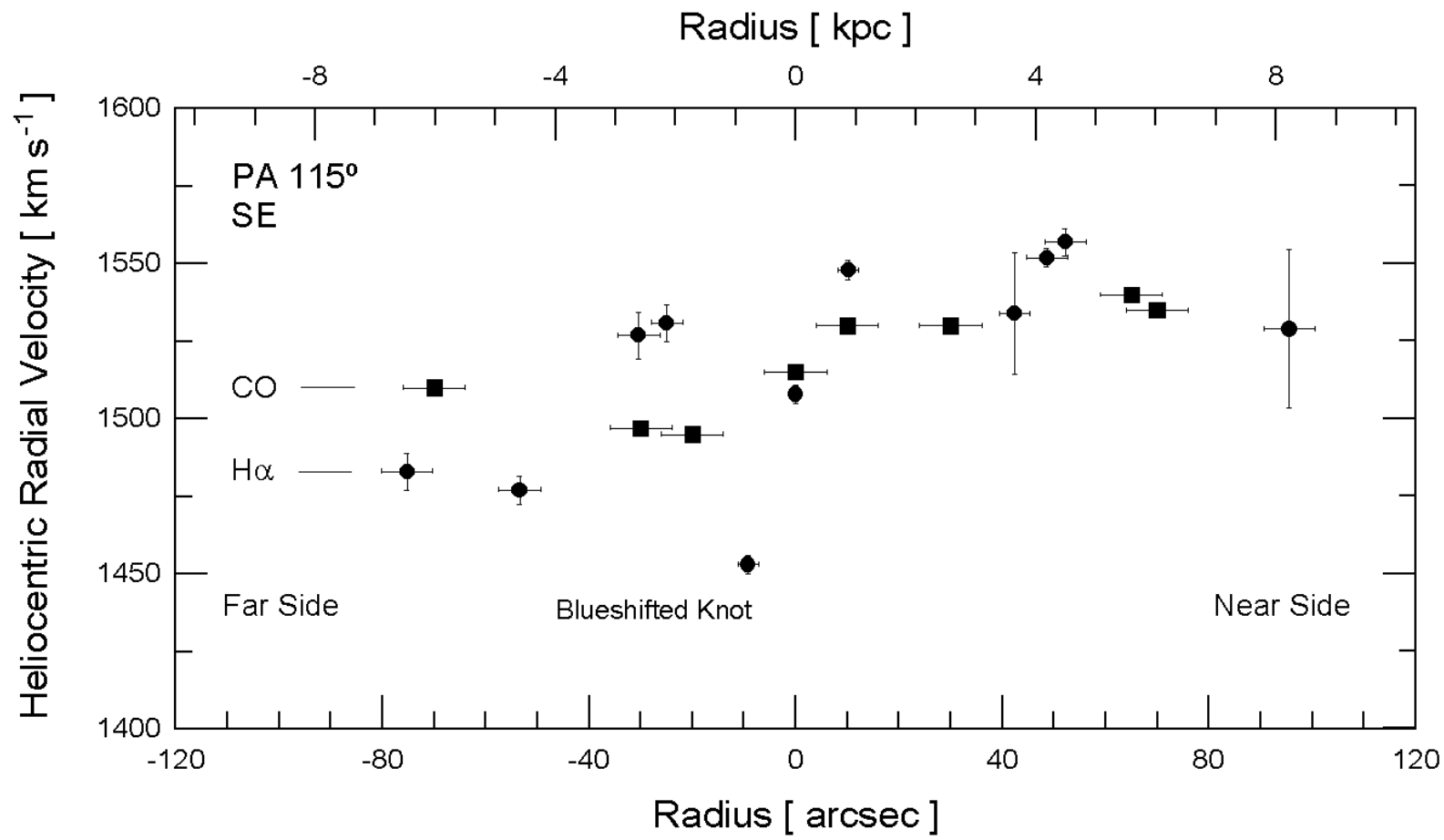

Fig. 3. Heliocentric radial velocities of NGC 1566 (from optical and radio data) along PA $=115^{\circ}$, a position angle near to the minor axis. The most conspicuous region with inflow motion is marked as "Blueshifted Knot".

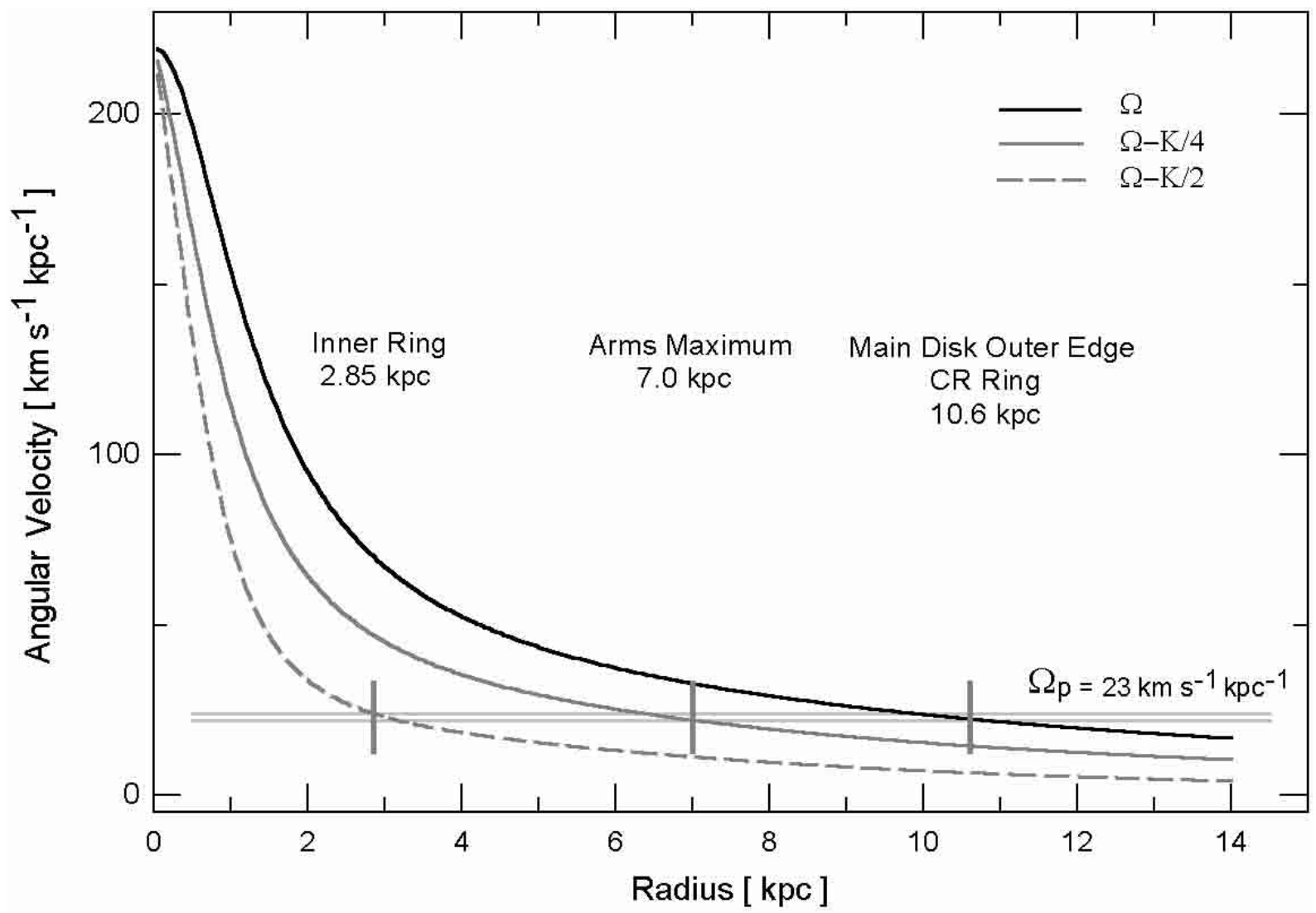

Fig. 4. Angular velocity curve corresponding to the fitted axial symmetric model. The Lindblad $\Omega-\kappa / 2$ and the ultra-harmonic $\Omega-\kappa / 4$ curves are also shown. Note that a pattern angular velocity of $(23 \pm 2) \mathrm{km} \mathrm{s}^{-1} \mathrm{kpc}^{-1}$ represents a unique solution that fits the main morphological features observed in $\mathrm{H} \alpha$ emission (Figs. 7-9). 
estimated the possible resonance radii, but they did not determined the pattern speed. Although their morphological analysis has large uncertainties, the suggestion that the corotation lies at the main disk outer edge is in agreement with our results. Vera-Villamizar et al. (2001) used three different numerical methods to analyze the grand design spiral galaxies NGC 1365, NGC 2997 and NGC 1566. The corotation radius determined for NGC 1566 by these authors is in agreement with our results, with the method based on a mode search with a twodimensional Fourier analysis (Puerari \& Dottori 1992) having the best agreement $(R \sim 10 \mathrm{kpc})$.

\subsection{Spectral emission}

The obtained spectra of NGC 1566 show strong emission lines in the nucleus and in the main body. Their intensities were derived by fitting Gaussian curves to their profiles. The nucleus presents narrow emission lines (Fig. 5). The fluxes derived from our observations are: $F\left(H \alpha_{\text {narrow }}\right)=$ $(1.10 \pm 0.05) \times 10^{-13} \mathrm{erg} \mathrm{cm}^{-2} \mathrm{~s}^{-1}, F(\lambda 6584)=(1.33 \pm 0.07) \times$ $10^{-13} \mathrm{erg} \mathrm{cm}^{-2} \mathrm{~s}^{-1}$, and $F(\lambda 6717+\lambda 6731)=(8.36 \pm 0.46) \times$ $10^{-14} \mathrm{erg} \mathrm{cm}^{-2} \mathrm{~s}^{-1}$. These fluxes are similar to those derived by other authors (e.g. Alloin et al. 1985). The $I(\lambda 6584) / I(\mathrm{H} \alpha)$ and $I(\lambda 6717+\lambda 6731) / I(\mathrm{H} \alpha)$ intensity ratios clearly indicate nuclear activity according to the diagnostic diagrams of Veilleux \& Osterbrock (1987). The $\mathrm{H} \alpha$ line shows also a broad component $(F W H M \approx 64 \AA)$ in the nuclear spectra, reflecting the Seyfert nature of NGC 1566 . The $\mathrm{H} \alpha$ narrow component $(F W H M \approx 5 \AA$ ) and the [N II] $\lambda \lambda 6548,6584$ lines are clearly seen superimposed on the broad $\mathrm{H} \alpha$ line. For comparison, Fig. 6 illustrates the spectrum of a zone located $74^{\prime \prime} \mathrm{SE}$ of the nucleus, typical of an emission region. On the other hand, the broad $\mathrm{H} \alpha$ component has been known to be variable for many years (Alloin et al. 1985) and as having sudden flux rises (e.g. Baribaud et al. 1992). The flux measured here $F\left(\mathrm{H} \alpha_{\text {broad }}\right) \approx(3.0 \pm 0.3) \times 10^{-13} \mathrm{erg} \mathrm{cm}^{-2} \mathrm{~s}^{-1}$ indicates that the nucleus was in a low level of activity during our observations. Its Doppler width in our spectra gives $F W H M \approx 2900 \mathrm{~km} \mathrm{~s}^{-1}$, a normal value for this kind of object.

The spectral characteristics of NGC 1566 have varied from a Seyfert 1.9 type galaxy during the minimum activity to a Seyfert 1.2 during the most active phases (Alloin et al. 1985; Winkler 1992). The shape of the $\mathrm{H} \alpha+[\mathrm{N} \mathrm{II}]$ lines is similar to that found by Alloin et al. (1985) between January and September 1981, with fluxes ranging from $(2.4 \pm 0.3) \times 10^{-13}$ to $(4.2 \pm 0.3) \times 10^{-13} \mathrm{erg} \mathrm{cm}^{-2} \mathrm{~s}^{-1}$, respectively. Since the Balmer decrement is not variable (Winkler 1992), those values and the one presented here imply that the object was in a stage of Seyfert 1.8 during our observations, because in the Alloin et al. spectra (1985) the $\mathrm{H} \beta$ component is visible for these $\mathrm{H} \alpha$ fluxes. We adopted the criteria of Winkler (1992), which are adapted from the Lick scheme (Osterbrock 1981).

Subtracting the continuum, the lines show that there are emission regions in the arms where the $\mathrm{H} \alpha$ fluxes are higher than or of the same order as in the nucleus, reflecting a very strong activity of star formation there. The $\mathrm{H} \alpha$ profiles, along all the observed directions, are plotted in Fig. 7 (where the distances were corrected using geometrical parameters fitted in Sect. 3.1). This plot also allows a qualitative estimation of the $S / N$ ratio along the different slit positions. In this figure the positions of the nuclear and inner rings (corresponding to the ellipses drawn in Fig. 8), of the arms maximum emission, and of a ring at the main outer edge are also indicated. Although there are no previous suggestions of a circumnuclear ring, most of these $\mathrm{H} \alpha$ profiles along the observed directions present a clear bump around the nucleus (Figs. 7 and 9). There is also evidence of an outer ring at $10.6 \mathrm{kpc}$ (more conspicuous in Fig. 7) that marks the transition to an outer region with looser faint spiral arms (Fig. 1). Its radial position would correspond to the corotation radius, as derived in Sect. 3.1. The star forming activity would be favoured by the significant amount of available gas, especially molecular, that the system has according to the CO observations (Bajaja et al. 1995). The H $\alpha$ luminosities, integrated over $8^{\prime \prime} .2 \times 2^{\prime \prime} .8(703 \mathrm{pc} \times 241 \mathrm{pc})$, corresponding to the bright regions located $74^{\prime \prime}$ southeast (along PA $=141^{\circ}$ ) and $76^{\prime \prime}$ northwest (along $\mathrm{PA}=157^{\circ}$ ) from the nucleus (Fig. 10) are respectively $L_{\mathrm{H} \alpha} \approx 3 \times 10^{6} L_{\odot}$ and $2 \times 10^{6} L_{\odot}$. These values imply that these arm sectors are populated by tens of $\mathrm{H}$ II regions with $\mathrm{H} \alpha$ luminosities in the upper limit of the most active star forming zones found by Rodrigues et al. $\left(1998, L_{\mathrm{H} \alpha} \leq 1.2 \times 10^{6} L_{\odot}\right)$ in the well studied spiral galaxy M94, assuming the same average size for the giant $\mathrm{H}$ II regions $\left(D_{0} \approx 115 \mathrm{pc}\right)$.

Figure 9 shows the $I(\lambda 6584) / I(\mathrm{H} \alpha)$ (black diamonds, being each of them the average of the five nearest values) and the $I(\lambda 6717+\lambda 6731) / I(\mathrm{H} \alpha)$ ratios (white diamonds) together with $\mathrm{H} \alpha$ flux along PA $=25^{\circ}$ (continuous line). In this figure the positions of the principal morphological features are also shown. The ratios are comparatively high in the central region of the galaxy; their decreasing trends are evident out of this region. The mean ratio errors are about $11 \%$ at the nucleus and less than $25 \%$ in the inter-arm zones. Assuming that those ratios depend mainly on the abundance effects, their radial distributions would indicate that the relative abundance of nitrogen and sulfur are about 3 times higher in the nucleus than in the intermediate zones $\left(90^{\prime \prime}\right)$. Furthermore, the $I(\lambda 6584) / I(\mathrm{H} \alpha)$ ratio decreases locally at a distance of 33" which corresponds to the ring at the bar end radius (inner ring), marked in Figs. 7 and 8. The high $\mathrm{H} \alpha$ flux there indicates important star formation activity, and the low $I(\lambda 6584)$ in relation to $\mathrm{H} \alpha$ suggests that the star bursts in that ring are younger than in the arms, as would be the case of the external regions at about 120" (corotation ring, Sect. 3.1) too.

The study of abundances in H II regions in the disks of spiral galaxies has shown the existence of negative gradients with a higher abundance towards the center of the galaxy (e.g. Pagel \& Edmunds 1981; Evans 1986; Shields 1990); NGC 1566 could be another example of this phenomenon. Our observations of the global trend and the differences between the arms, inter arms and rings regions of the $I(\lambda 6584) / I(\mathrm{H} \alpha)$ ratio are consistent with the local study of the NW arm of NGC 1566 made by Roy \& Walsh (1986), in which they found spatial variations in the metal abundance index on scales as small as $100 \mathrm{pc}$.

The distributions corresponding to $\mathrm{PA}=141^{\circ}$ and $157^{\circ}$ of the $\mathrm{H} \alpha$ flux densities (our results), the continuum at $843 \mathrm{MHz}$ 


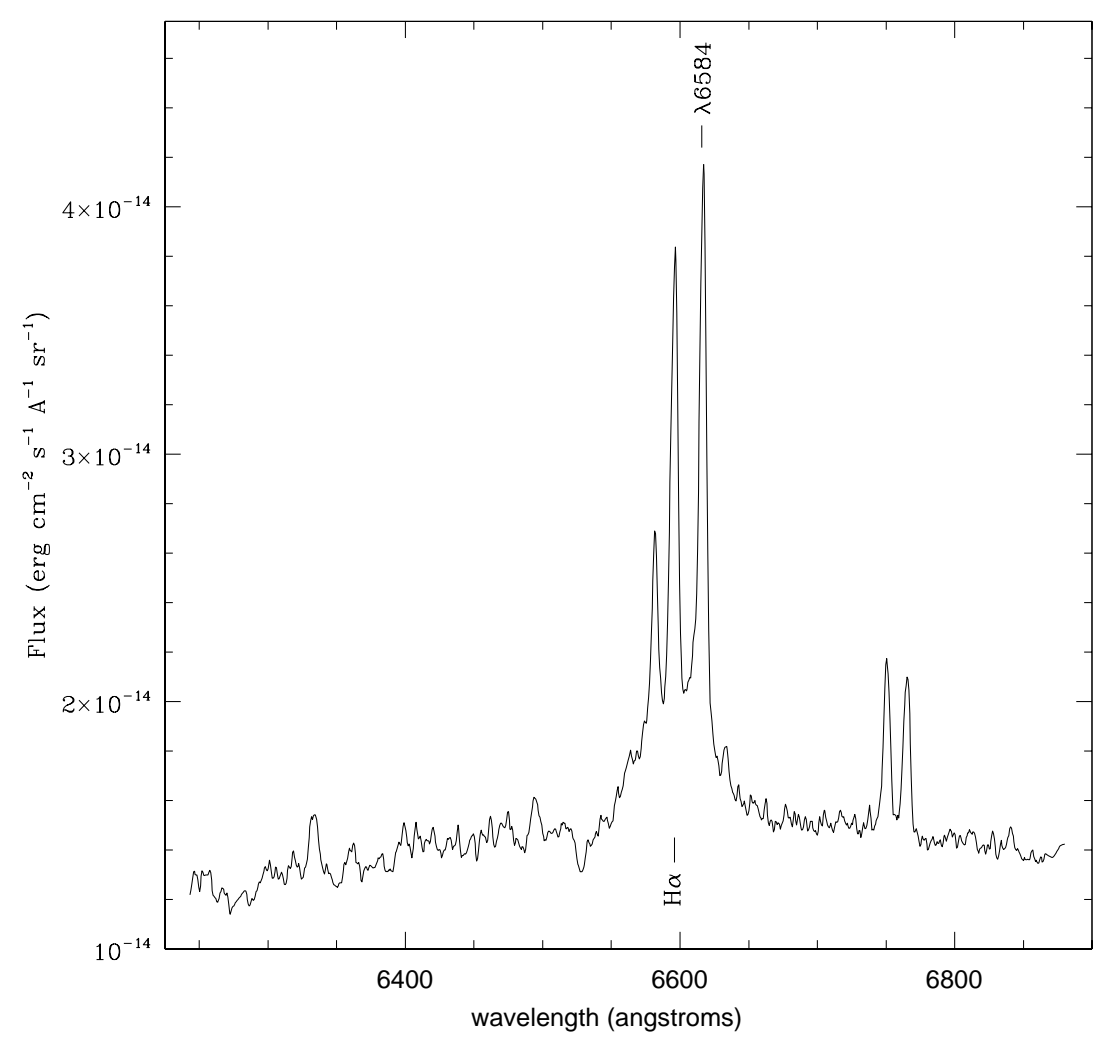

Fig. 5. Nuclear spectrum of NGC 1566. Dispersion is $32 \AA \mathrm{mm}^{-1}$. The presence of the broad component of the H $\alpha$ line at the time of observation can be compared with the spectra shown by Alloin et al. (1985).

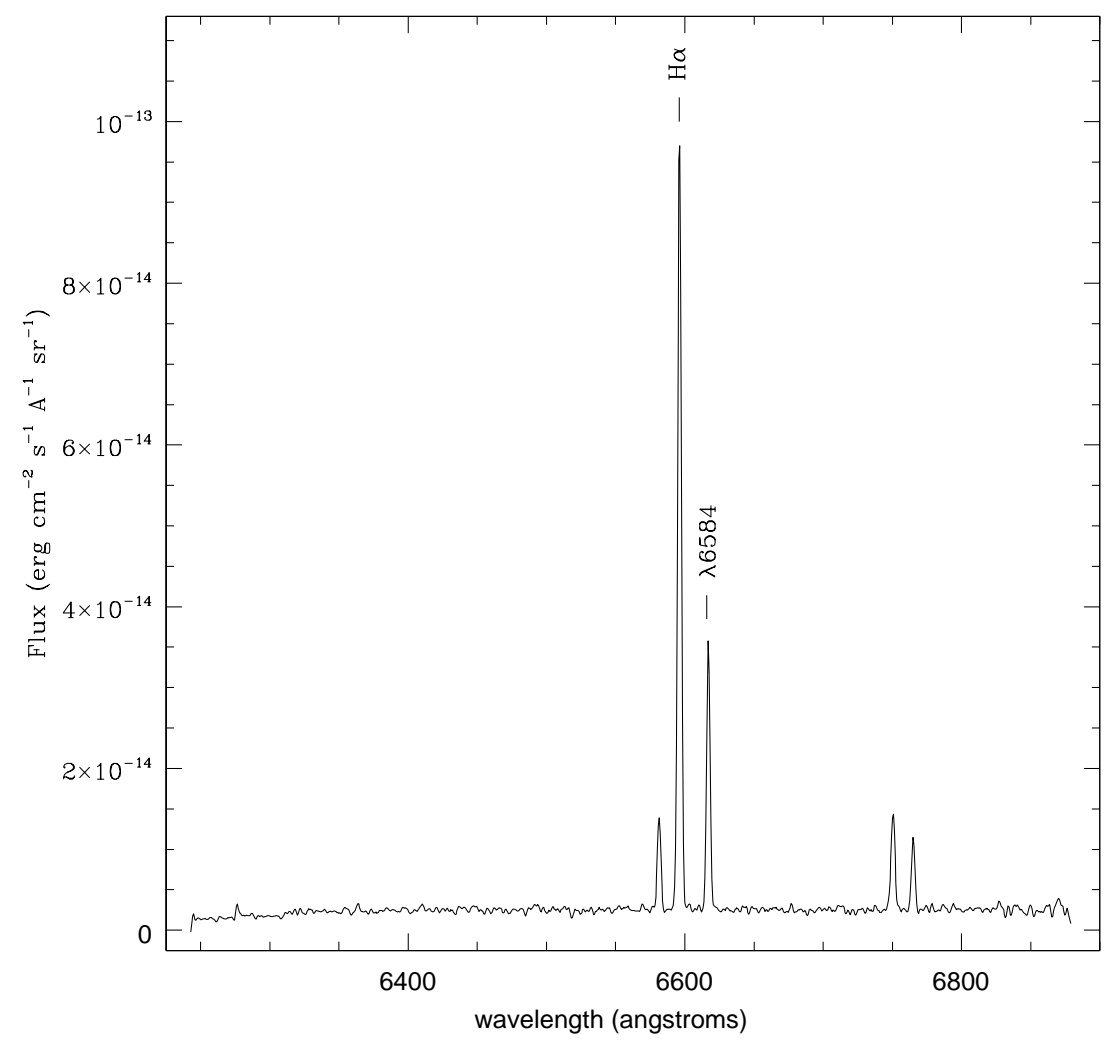

Fig. 6. Spectrum of the emission region located at $74^{\prime \prime} \mathrm{SE}$ of the nucleus along PA $=141^{\circ}$. Dispersion is $32 \AA \mathrm{mm}^{-1}$. 


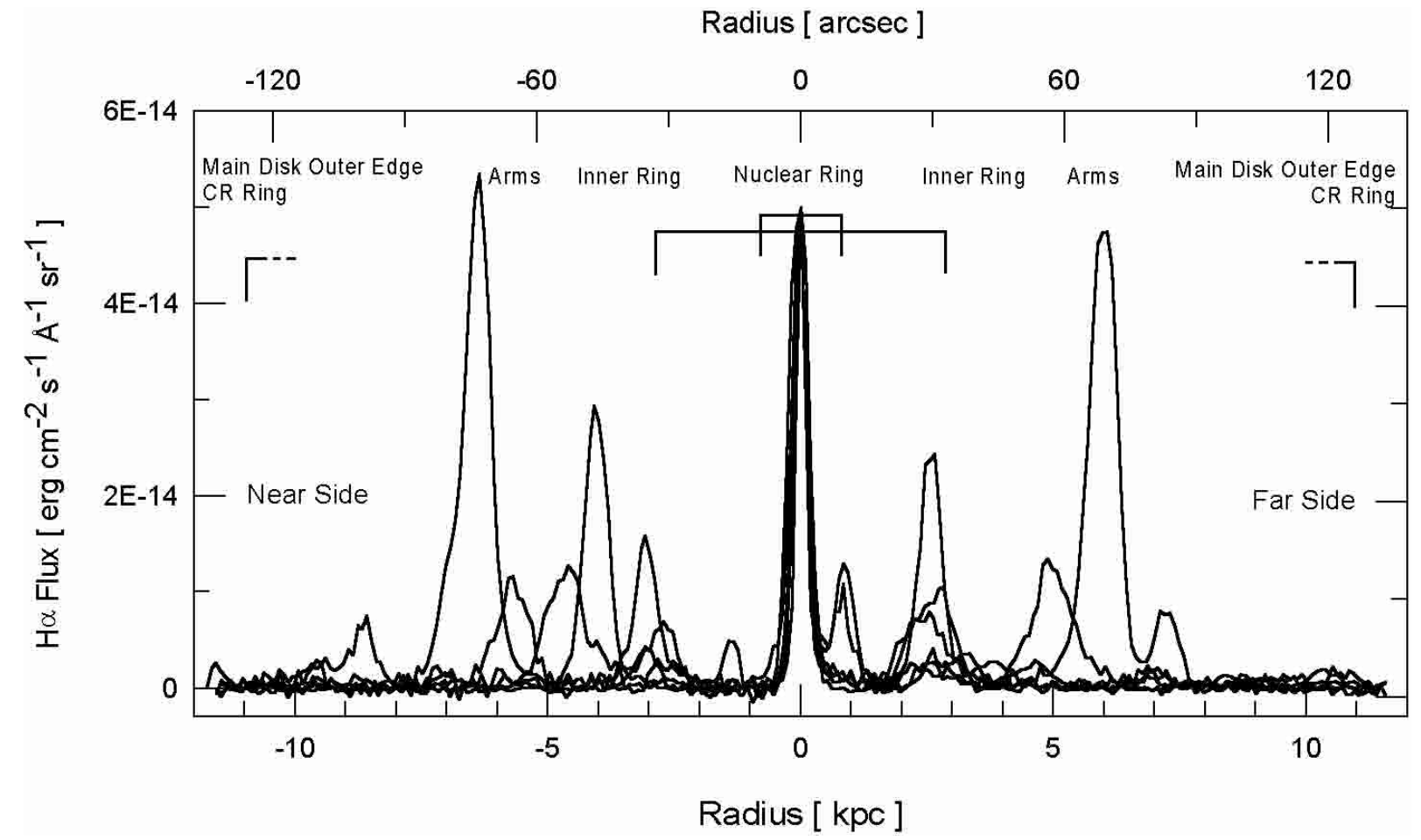

Fig. 7. $\mathrm{H} \alpha$ flux distributions along the six observed directions. The distances from the nucleus are corrected by inclination and position angles. The principal morphological features are marked.

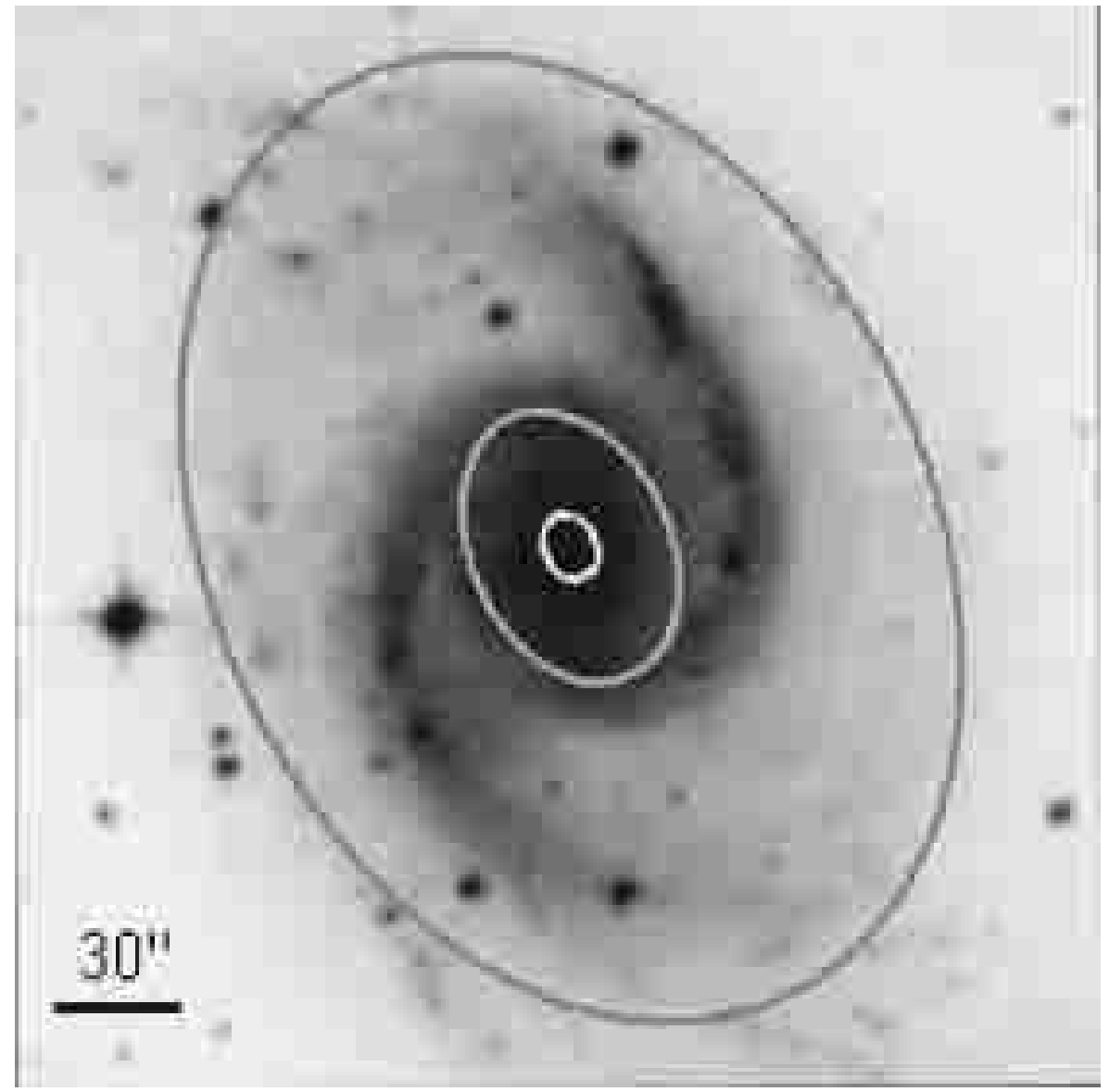

Fig. 8. Ellipses representing the nuclear, inner, and corotation rings marked in Figs. 7 and 9, superimposed on a blue optical image of NGC 1566 (from ESO plates). 


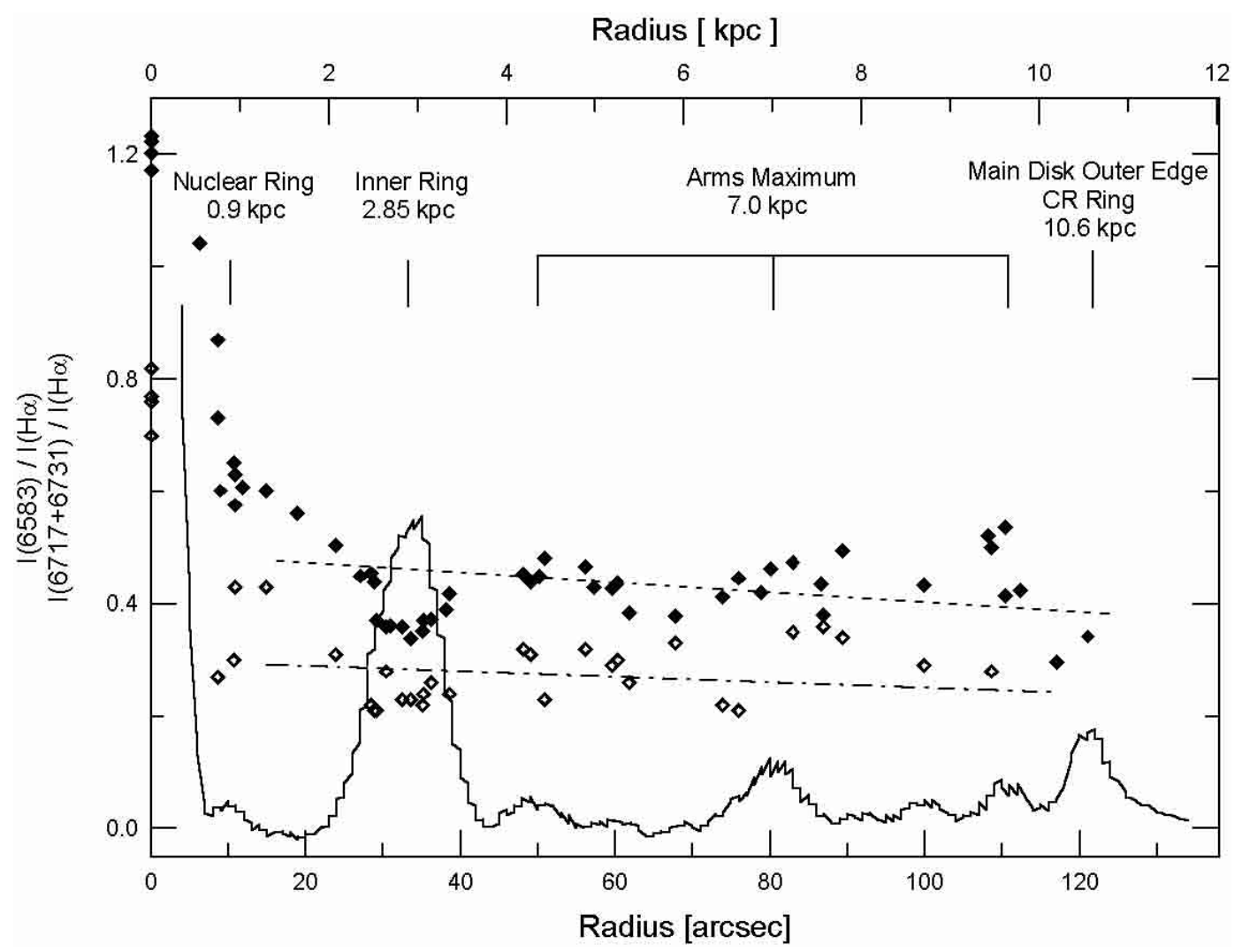

Fig. 9. Radial distribution of the $I(\lambda 6584) / I(\mathrm{H} \alpha)$ (black diamonds) and $I(\lambda 6717+\lambda 6731) / I(\mathrm{H} \alpha))$ (white diamonds) ratios, and the $\mathrm{H} \alpha$ flux along $\mathrm{PA}=25^{\circ}$ (continuous line). The dashed lines (both types of strokes) represent mean least square interpolations between the respective values out of the central region. The distances from the nucleus are corrected by inclination and position angles.

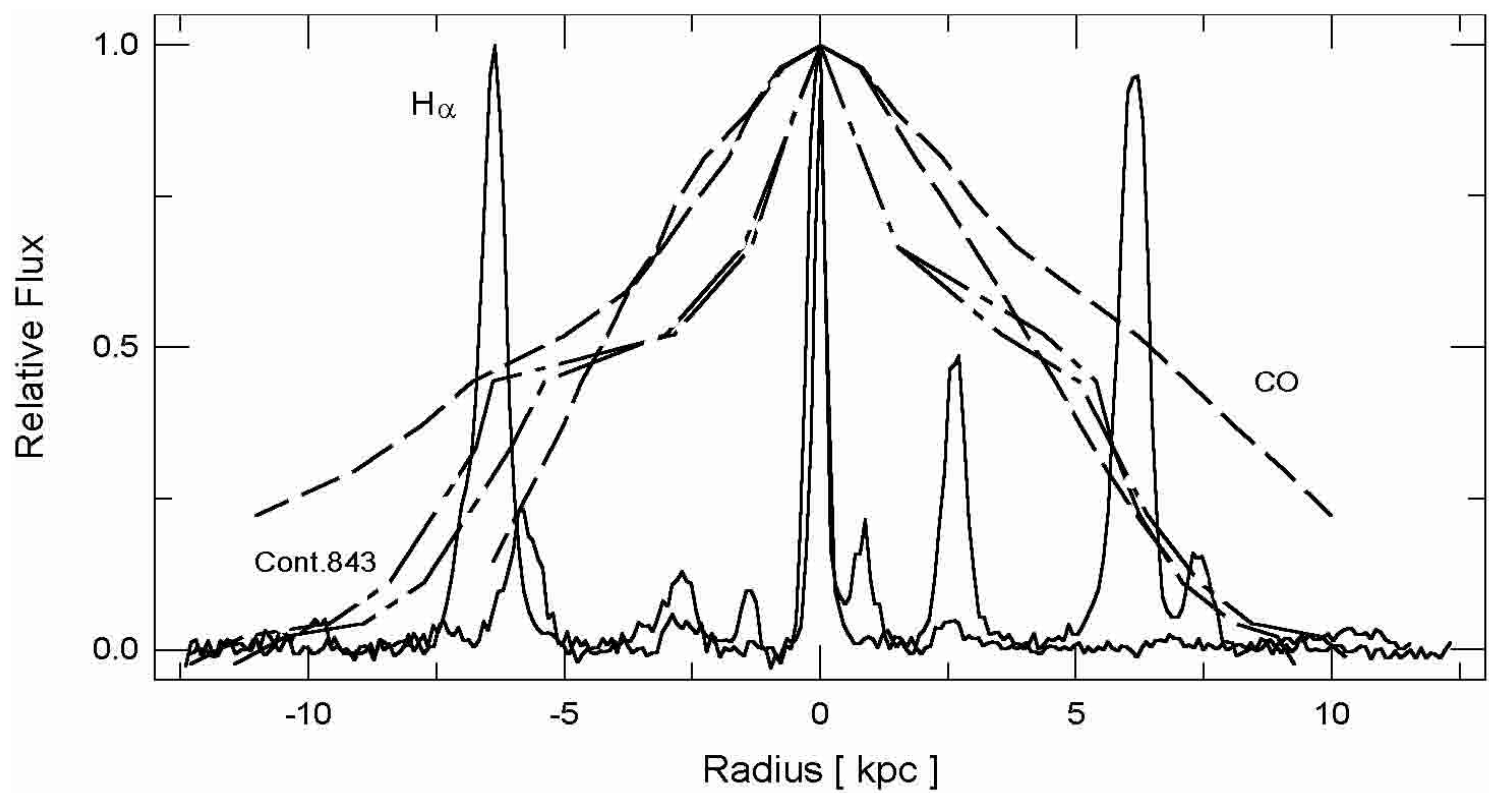

Fig. 10. Intensity radial distributions along $\mathrm{PA}=141^{\circ}$ and $\mathrm{PA}=157^{\circ}$ for the $\mathrm{CO}$ (Bajaja et al. 1995) at $115 \mathrm{GHz}$ and $\mathrm{H} \alpha$ (this paper) lines, and for the continuum at $843 \mathrm{MHz}$ (Harnett 1984).

(Harnett 1984), and the CO emission line at $115 \mathrm{GHz}$ (Bajaja et al. 1995) as a function of the distance to the center and normalized to the center, are illustrated in Fig. 10. In spite of the different angular resolutions, which are evident in the widths of the central peaks, a very good general correlation between the three curves is observed (see also arm locations in Fig. 7). This correlation suggests that $\mathrm{H} \alpha$ emission would be related to the thermal component of the continuum radiation and the molecular gas over the whole galaxy. 


\section{Conclusions}

From the spectrophotometrical and kinematical study of the central region and main body of NGC 1566 we found that:

- The Satoh fitted model leads to a total mass of $2 \times$ $10^{11} M_{\odot}$, which is coincident with the one obtained from the H I global velocity profile. This implies that the rotation curve is well represented by the fitted two-component model, without the need for a massive halo component.

- Considering the rotation curve of the fitted model and assuming a trailing spiral pattern, an outflow motion related to the bar is detected here.

- Other non-circular motions are observed in some regions, with a maximum significant blue shift near to the minor axis reaching a value of about $60 \mathrm{~km} \mathrm{~s}^{-1}$. From the location of these regions, and assuming that those motions take place on the galactic plane, they would represent inflows of gas toward the nucleus. These inflows could constitute the feeding of the nucleus of NGC 1566, the small bar mentioned above being the only deviation observed from the axial symmetry.

- A pattern angular velocity of $(23 \pm 2) \mathrm{km} \mathrm{s}^{-1} \mathrm{kpc}^{-1}$ is a unique solution that fits the radial positions of the inner ring, the star forming ridge of the arms, and the main disk outer edge. The corotation resonance should be located at $10.6 \mathrm{kpc}$, the radius of the ring corresponding to the main disk outer edge that marks the transition to an outer region with fainter spiral arms.

- The nucleus was in a stage of Seyfert 1.8 and in a low level of activity during observations.

- Circumnuclear and inner rings were detected, the diameter of the latter being nearly equal to the length of the small bar seen in the central region. There is also evidence of a ring at the main disk outer edge, pointing out the transition to a different morphological region, the outer disk region with looser faint spiral arms.

- Some emission regions in the arms have $\mathrm{H} \alpha$ fluxes higher than that of the nucleus.

- The star bursts in the ring at the bar end radius would be younger than in the arms.

- There is a very good correlation between the radial distributions of the $\mathrm{H} \alpha$, the continuum at $843 \mathrm{MHz}$, and the $\mathrm{CO}$ line at $115 \mathrm{GHz}$, suggesting that the $\mathrm{H} \alpha$ emission is related to the thermal component of the continuum radiation and the molecular gas over the whole galaxy.
Acknowledgements. RD acknowledges useful discussions with Horacio Dottori about NGC 1566 and dynamical resonances in galaxies.

\section{References}

Alloin, D., Pelat, D., Phillips, M., \& Whittle, M. 1985, ApJ, 288, 205

Athanassoula, E. 1992, MNRAS, 259, 345

Bajaja, E., Wielebinski, R., Reuter, H. P., Harnett, J. I., \& Hummel, E. 1995, A\&AS, 114, 147

Baribaud, T., Alloin, D., Glass, I., \& Pelat, D. 1992, A\&A, 256, 375

Becker, R., Mebold, U., Reif, K., \& van Woerden, H. 1988, A\&A, 203, 21

Beckmann, J., Bransgrove, S., \& Phillips, J. 1986, A\&A, 157, 49

Binney, J., \& Tremaine, S. 1994, in Galactic Dynamics (Princeton Univ. Press), 44

Combes, F. 2001, Galaxy Disks and Disk Galaxies, ed. J. G. Funes, \& E. Corsini, ASP Conf. Ser., 230, 213

Elmergreen, B. G., \& Elmergreen, D. M. 1990, ApJ, 355, 52

Evans, I. 1986, ApJ, 309, 544

Harnett, J. I. 1984, MNRAS, 210, 13

Malkan, M., Gorjian, V., \& Tam, R. 1998, ApJS, 289, 81

Osterbrock, D. 1981, ApJ, 249, 462

Pagel, B., \& Edmunds, M. 1981, ARA\&A, 19, 77

Puerari, I., \& Dottori, H. 1992, A\&AS, 93, 469

Piner, B., Stone, J., \& Teuben, P. 1995, ApJ, 449, 508

Reif, K., Mebold, U., Gross, W., van Woerden, H., \& Siegman, B. 1982, A\&AS, 50, 451

Rodrigues, I., Dottori, H., Cepa, J., \& Vilchez, J. 1998, A\&A, 128, 545

Roy, J., \& Walsh, J. 1986, MNRAS, 223, 39

Rubin, V., Burstein, D., Ford, W., \& Thonnard, N. 1985, ApJ, 289, 81

Shields, G. 1990, ARA\&A, 28, 525

Stone, R. P. S., \& Baldwin, J. A. 1982, MNRAS, 204, 347

Tully, R. 1988, Nearby Galaxies Catalog (Cambridge Univ. Press)

Veilleux, S., \& Osterbrock, D. 1987, ApJS, 63, 295

Vera-Villamizar, N., Dottori, H., Puerari, I., \& de Carvalho, R. 2001, ApJ, 547, 187

Winkler, H. 1992, MNRAS, 257, 677 